\title{
Estudiantes universitarios ante los retos formativos de las Redes Sociales: el caso de Colombia
}

\author{
José Manuel PÉREz TORNERO \\ josepmanuel.perez@uab.cat \\ Universitat Autònoma de Barcelona \\ Santiago Tejedor CALvo \\ santiago.tejedor@uab.cat \\ Universitat Autònoma de Barcelona \\ Núria Simelio SolÀ \\ nuria.simelio.sola@uab.cat \\ Universitat Autònoma de Barcelona \\ Beatriz Elena MARÍN OCHOA \\ beatrize.marin@upb.edu.co \\ Universidad Pontificia Bolivariana (Colombia)
}

Recibido: 10 de febrero de 2014

Aceptado: 9 de septiembre de 2014

\section{Resumen}

Este artículo analiza los retos formativos de estudiantes universitarios colombianos de Ciencias de la Comunicación con el objetivo de diagnosticar su uso de las redes sociales. Se han realizado encuestas a 71 estudiantes y 12 grupos focales. Los resultados muestran que los estudiantes analizados utilizan las redes sociales como herramientas complejas que les que les permiten formarse, participar, informarse, cooperar, trabajar y entretenerse. Los estudiantes demuestran un alto nivel crítico y de conocimiento de las ventajas y desventajas de las redes. Las conclusiones muestran que las redes sociales son un reto formativo para las nuevas generaciones de estudiantes de Comunicación ya que su practicidad, inmediatez y eficacia, las convierten en una fuente periodística prioritaria, que debe ser también tenida en cuenta por los formadores de los futuros periodistas.

Palabras clave: Redes sociales, Universidad, Uso Pedagógico, Comunicación, Internet.

\section{University Students Faced with the Formative Challenges of Social Networks: Colombian Case Study}

\begin{abstract}
This article analyses the formative challenges of Colombian University Students from the Faculty of Communication Studies. The principal aim of this research is to identify their use of the social networks. Seventy-one surveys and 12 focus groups were carried out. The results show that students in the sample use the social networks as complex tools that allow them to train, inform, participate, cooperate, work and entertain themselves. The students demonstrate a high critical level and an important knowledge of the advantage and disadvantages of the networks. The conclusions demonstrate that social networks are a formative challenge for the new generations of Communication students. Their effectiveness, immediacy and efficiency make them a fundamental journalistic source that needs to be taken into account by faculty of future journalists.

Keywords: Social Networks, University, Educational Use, Communication, Internet.

\section{Referencia normalizada}

PÉREZ TORNERO, José Manuel; TEJEDOR CALVO, Santiago; SIMELIO SOLÀ, Núria; y MARÍN OCHOA, Beatriz Elena (2015): "Estudiantes universitarios ante los retos formativos de las Redes Sociales: el caso de Colombia". Estudios sobre el Mensaje Periodístico. Vol. 21, Núm. 1 (enero-junio), págs.: 509-521. Madrid, Servicio de Publicaciones de la Universidad Complutense.
\end{abstract}


Sumario: 1. Introducción. Los futuros comunicadores ante las redes sociales; 1.1. Participación, cooperación y Redes Sociales; 1.2. Contexto: Colombia. 2. Material y métodos. 3. Análisis y resultados. 4. Discusión y conclusiones 5. Referencias bibliográficas.

\section{Introducción. Los futuros comunicadores ante las redes sociales}

El crecimiento de las plataformas de la Web 2.0 ha sido exponencial en los últimos años. La filosofía colaborativa de la web social se ha expandido a todos los escenarios de nuestro día a día: desde el ámbito laboral hasta el más personal o cotidiano. Al mismo tiempo, que constituye un escenario natural de aprendizaje tanto formal como informal (Dabbagh y Kitsantas, 2012). En el caso concreto de la comunicación, el papel de Internet y, especialmente, de las redes sociales, ha adquirido un protagonismo destacado. En este sentido, los medios de comunicación (tanto analógicos como digitales) se han apresurado en crear sus plataformas $2.0 \mathrm{y}$ en utilizarlas como canales de información, de comunicación y de marketing de sus contenidos. Este conjunto de cambios ha generado nuevas pautas de consumo, acceso y manejo de la información. (Túñez, et al., 2010) Si bien el número de visitantes de los cibermedios se ha mantenido estable, la cantidad de usuarios de las redes sociales se ha duplicado en los últimos años, siendo Facebook y Twitter los casos más representativos. (The Cocktail Analysis, 2013). Este conjunto de transformaciones introduce importantes cambios en la concepción que los nativos digitales -futuros profesionales de la comunicaciónposeen de los medios de comunicación y en el tipo de usos que realizan de las redes sociales. (WAYCOTT, et al., 2010). Ambos aspectos exigen cambios imprescindibles en el escenario académico para garantizar una formación adaptada a las nuevas necesidades formativas que introduce la red de redes. Se trata de modificaciones que afectan tanto a los profesores como a los docentes. (Roblyer, et al., 2010).

En este escenario, la investigación «Estudiantes ante los retos formativos de la Sociedad Red. Nivel de conocimiento, habilidades técnicas y tipologías de uso de las plataformas 2.0: El caso de Colombia» tuvo como principal objetivo llevar a cabo un estudio diagnóstico del nivel de dominio, habilidades y competencias -así como el tipo de usos y aplicaciones- de las plataformas 2.0 que poseen los alumnos del ámbito de Comunicación de la Universidad Pontificia Bolivariana de Colombia (UPB).

Los objetivos generales del de la investigación que presentamos se pueden sintetizar en los siguientes puntos:

Realizar un análisis diagnóstico general sobre el uso de las Tecnologías de la Información y la Comunicación (TIC) entre estudiantes de Comunicación de la Pontificia Universidad Bolivariana de Medellín, Colombia, (UPB).

Establecer qué tipo de herramientas y plataformas 2.0, y específicamente redes sociales, utilizan en mayor medida los estudiantes de Comunicación de la UPB.

Detectar la tipología de usos que los estudiantes de Comunicación de la UPB realizan de las redes sociales, atendiendo específicamente a su uso como herramienta formativa.

Establecer el valor informativo (fiabilidad, credibilidad, calidad, etc.) que los alumnos de Comunicación de la UPB confieren a las redes sociales y de información (especialmente, Facebook y Twitter). 


\subsection{Participación, cooperación y Redes Sociales}

La Web 2.0 (O'Reilly, 2007) se concibe como un espacio para la generación compartida de conocimientos, para el trabajo cooperativo y para la publicación a escala universal de todo tipo de contenidos (textos, imágenes, sonidos y vídeos). Diversos autores han estudiado el nuevo concepto de audiencia activa (Jenkins, 2008; Harrison y Barthel, 2009) y como este conjunto de cambios posibilita que cualquier usuario pueda convertirse en un emisor de contenidos dotado de herramientas que le permitan difundir contenidos por toda la Red, superando las barreras del espacio y del tiempo. Así, sin disponer de un conocimiento técnico, y haciendo uso de herramientas gratuitas que aporta la Red, el internauta puede actuar como un emisor de contenidos en el mismo nivel que los periodistas y/o medios de comunicación. Este aspecto ha sido presentado por los investigadores (Thurman, 2008; Lewis, et al., 2009) como un cambio radical en los procesos de difusión, distribución y acceso a la información. La denominada web social o web 2.0 (ya superada por la denominada Web 3.0) se resume en el paso de las tradicionales web a otras destinadas a los usuarios. De la concepción estática de los sitios web se evoluciona a otros marcados por un cambio constante que, en muchas ocasiones, procede tanto de los gestores del mismo como de los mismos usuarios, que adquieren los roles de editores e incluso generadores contenidos. (Allan y Thorsen, 2009).

Según un estudio del «Pew Research Center's Internet American Life Project» y el «Project for Excellence in Journalism» (Purcell, et al., 2010) Internet y las redes sociales han convertido las informaciones en portátiles, personalizadas y participativas. En este nuevo ecosistema multiplataforma, los autores destacan como un $33 \%$ de usuarios de teléfonos móviles accede a las noticias a través de ellos y que un $37 \%$ de los usuarios de Internet ha contribuido a la creación de noticias, ha comentado informaciones o las ha difundido por redes sociales como Facebook y Twitter. (Purcell, et al., 2010: 2).

Además, el estudio añade que la fidelidad de la audiencia es cada vez más frágil. Así, el 46\% de los estadounidenses afirma que consume noticias de cuatro a seis medios de comunicación. Apenas el 7\% obtiene información de una única fuente. Seis de cada diez estadounidenses (59\%) recibe noticias de una combinación de fuentes en línea y fuera de línea. Por otro lado, en la investigación se sostiene que Internet ha generado estos cambios en la audiencia. De manera específica se señala que las redes sociales y los blogs han transformado el consumo de noticias en una experiencia social. (Purcell, et al., 2010: 2).

Por otro lado, en este estudio, Purcell, et al. (2010), introducen ya un conjunto de cambios de gran interés en la investigación de la comunicación digital y, especialmente, sobre los nuevos tipos de usos y la credibilidad conferida a estas nuevas plataformas colaborativas. Los autores coinciden en señalar que EEUU es el país que ha llevado a cabo un mayor número de investigaciones sobre periodismo participativo desde diferentes enfoques y perspectivas. Tal y como apuntan Palacios y Díaz Noci (2007) la investigación empírica sobre el periodismo ciudadano es aún incipiente:

« [...] las aproximaciones metodológicas son muy diversas, buscando la perspectiva más adecuada para abordar el fenómeno: análisis estructural de las oportunidades de par- 
ticipación que ofrecen las webs de medios convencionales, análisis de contenido de material 'periodístico' producido por los ciudadanos; entrevistas con periodistas, promotores de medios ciudadanos y ciudadanos mismos para entender sus actitudes y motivaciones». (Palacios y Díaz Noci, 2007: 85)

Con relación a la reflexión de estos autores, cabe señalar que la presente investigación es un estudio diagnóstico sobre el nivel de conocimiento, y sobre las tipologías de usos que los estudiantes de Comunicación de la UPB de Medellín realizan de la plataformas 2.0, y específicamente de las redes sociales.

\subsection{Contexto: Colombia}

«Medellín, la ciudad más innovadora del mundo» fue el titular con el que los colombianos se sorprendieron el 1 de marzo de 2013, cuando en los principales medios de esta ciudad colombiana se registró en primera página la noticia. La ciudad había sido elegida en el marco del concurso "City of the Year», que organizan The Wall Street Journal y Citigroup. Había competido con Nueva York y Tel Aviv y tras la decisión del resultado de una votación por Internet los organizadores valoraban su proceso de desarrollo que incluía la reducción de emisiones de $\mathrm{CO} 2$, la reducción de la criminalidad y la creación de espacios culturales.

La ciudad que hasta hace unos años era conocida por ser una de las más violentas del mundo, ahora se convertía en protagonista de buenas noticias, porque en efecto Medellín como otras ciudades de Colombia son de contrastes. El país por ejemplo pasa en la actualidad por un buen momento económico, político y social; y aunque es observada con prevención desde fuera porque ha enfrentado en los últimos 50 años fenómenos como: la violencia, el narcotráfico, la guerrilla, los paramilitares y la corrupción, demostró que es posible salir adelante en los momentos más difíciles, de ahí que no es extraño que aun siendo un país con un crecimiento de población constante que en 2012 alcanzó los 47,7 millones de ciudadanos, según cifras del Banco Mundial $^{1}$ (2013); de poseer una cifra que no logra bajar el índice de 90\% en materia alfabetización (UIS FACT SHEET, 2013: 2); de contar con un índice de lectura de 2 libros por año según el estudio adelantado por el Departamento Administrativo Nacional de Estadística - DANE y su encuesta cultural (DANE:2013,9), y de estar en el rango de los países que según el informe de Medición de la Unión internacional de Telecomunicaciones, ITU, (DANE: 2012: 2): “Se prevé que la tasa de penetración de la banda ancha móvil siga creciendo a una tasa de dos dígitos durante los próximos años, como reflejo de la expansión de los servicios de Internet móvil», lo que le permite poseer redes de telefonía con casi dos líneas por habitante que lo ubican como uno de los países con más desarrollo a nivel de conectividad, redes, usos y por supuesto alfabetización digital.

1 Cifras actualizadas de población del Banco Mundial a noviembre de 2013, pueden encontrarse en el apartado de Datos Colombia y está disponible en la web: http://datos.bancomundial.org/pais/colombia 
Es por ello que programas locales como Medellín Digital ${ }^{2}$, regionales como Antioquia Digital ${ }^{3}$ o de país como el Plan Vive Digital ${ }^{4}$ han propiciado avances en las comunidades que hoy se apropian de las redes, participan en ellas, desarrollan nuevos contenidos, diseñan estrategias de conectividad y contribuyen activamente al trabajo colaborativo. Y es que desde que Internet llegó a Colombia en 1994, gracias a la Red de Ciencia, Educación y Tecnología de Colombia (CETCOL), permitió, más allá del acceso inmediato a la información, nuevas formas de interacción entre los ciberciudadanos y entre ellos los jóvenes quienes se distinguieron por ser desde el comienzo los protagonistas.

Un protagonismo que sigue ahí pues su necesidad de conexión crece día a día con cada nuevo dispositivo o APP que surge en el mercado, según el último informe emitido por el MinTic: El crecimiento de las conexiones a Internet en los dos últimos años supero el 180\% (MINTIC, 2013: 24).

Esto se evidencia en los 17.000.000 de usuarios que reseñaba El Tiempo (2013) al anunciar el establecimiento de una sede de Facebook en el país y agregar que de ellos 4,266.810 eran jóvenes entre los 18 y los 24 años, lo que equivale al 34\%; seguido del $26 \%$ perteneciente al rango edad de 25 a 34 años. Y el de la red informativa Twitter que alcanzaba los seis millones de usuarios (Castrillón, 2013: 22).

Todo esto hace del país un líder en conectividad en América Latina, según el informe del MinTic «Hay 325 municipios conectados con fibra óptica, y con la adjudicación del contrato se va a llegar 1.078 municipios (96\% del país). Los otros 44 municipios tendrán conectividad de alta velocidad con otra alternativa. La meta es que en 2014 todos los municipios estén conectados» (MINTIC, 2011).

Por tanto, el estudio se produce en un contexto en el que se ha dado un incremento del tiempo que los usuarios dedican al uso de las diferentes redes sociales de naturaleza generalista que existen en Internet. Este crecimiento invita a analizar cómo los estudiantes de Comunicación -que accederán en unos años al mercado laboral- conciben, utilizan y conocen las redes sociales y otras plataformas que han emergido con la Web 2.0.

2 Medellín Digital hoy denominado Medellín inteligente, es un programa liderado por la Alcaldía de Medellín, con el objetivo de fomentar y facilitar el buen uso de las Tecnologías de información y comunicación en la ciudad. Más información está disponible en: http://www.medellindigital.gov.co/mediateca/Paginas/Inicio.aspx

3 Antioquia Digital es un programa que busca la apropiación y uso de las Tecnologías de la Información y las Comunicaciones (TIC), como una herramienta que facilita el mejoramiento de la calidad educativa en el departamento de Antioquia, a través de la implementación de 350 Colegios Digitales, el desarrollo y uso de un Metaportal Educativo y acceso a Internet del $80 \%$ de los establecimientos educativos oficiales la información está disponible en: http://www.antioquiadigital.edu.co/

4 Plan Vive Digital de Colombia es el plan de tecnología para los próximos cuatro años en Colombia, que busca que el país dé un gran salto tecnológico mediante la masificación de Internet y el desarrollo del ecosistema digital nacional. Más información disponible en: http://www.mintic.gov.co/index.php/vive-digital. 


\section{Material y métodos}

El estudio se llevó a cabo en el marco del curso académico 2013-2014 con un total de 71 estudiantes (de ambos sexos; con edades comprendidas entre los 18 y los 23 años; y cursando estudios de comunicación en diferentes cursos o niveles) en la Facultad de Comunicación Social- Periodismo de la Universidad Pontificia Bolivariana (UPB) de Medellín (Colombia). Esta facultad acaba de cumplir 45 años y está acreditada por el Ministerio de Educación Nacional de Colombia. Además, la Universidad cuenta con acreditación internacional y está considerada como una de las 12 mejores universidades del país. El último Ranking Sapiens, reporte 6, la ubica en el cuarto lugar entre las universidades privadas y en el 11 al contabilizar también las públicas ${ }^{5}$.

La facultad según comentar su director el Esp. Juan Fernando Muñoz Uribe cuenta en la actualidad con alrededor de 1200 estudiantes, la mayoría provenientes de estratos socioeconómicos medios y altos (3, 4 y 5), seguido en segundo lugar por los de estratos bajos y muy altos (1, 2 y 6 ). Aunque es importante resaltar que el rango del estrato 3 se ha venido ampliando en los últimos años debido a las múltiples posibilidades de ingreso que en materia de becas y apoyos ofrece la Universidad a sus aspirantes.

La investigación se ha basado en una metodología de trabajo híbrida que cruza el enfoque cuantitativo con el cualitativo, utilizando las metodologías más adecuadas para este tipo de estudios como son las encuestas y los grupos de discusión. (Gómez et al., 2012). A través de cuestionarios cerrados se ha intentado establecer el nivel de conocimiento, el tipo de uso y la importancia que los estudiantes de Comunicación confieren a la Web 2.0 y a las redes sociales en la formación de un comunicador. Se han aplicado diferentes cuestionarios, así como el cruce de resultados de éstos y la generación de nuevos cuestionarios a partir de los resultados obtenidos. Los datos cuantitativos se han analizado a partir de frecuencias y porcentajes.

Las variables principales de las preguntas de los cuestionarios han sido: a) Presencia en redes sociales. b) Tipología y características de las redes sociales. c) Motivaciones en el uso de las redes sociales. d) Tiempo dedicado a las redes sociales. e) Transformaciones cotidianas generadas por las redes sociales en su quehacer académico, personal y profesional.

Además, se han llevado a cabo 12 grupos focales con los mismos estudiantes, procedentes de los diferentes cursos que integran la carrera de Comunicación Social. Estos espacios de encuentros han servido para profundizar en la concepción, la aplicación y el tipo de usos que los estudiantes de Comunicación realizan de las plataformas de la Web 2.0. Los grupos focales tuvieron una duración de 100 a 120 minutos de duración y fueron guiados por un moderador que formaba parte de este proyecto de investigación. Los principales ítems del guión de los grupos focales han sido: a) Explicar qué es una red social. b) Reflexionar sobre las fortalezas y debilidades de las

\footnotetext{
5 El Ranking Sapiens es un informe que presenta el Sapiens Research Group y que se ofrece con el Boletín Científico Sapiens Research. Se creó en el año 2010 con ISSN-e 2215-9312 y lanzó su primer número electrónico-digital en enero de 2011. Disponible en: http://www.sapiensresearch.org/ranking/u-sapiens.
} 
redes sociales. c) Exponer las motivaciones por las que tener presencia en una red social. d) Debatir sobre el tipo de uso y el tiempo dedicado a las redes sociales. e) Reflexionar sobre los aportes de las redes sociales en su formación y desempeño profesional como profesionales de la comunicación.

\section{Análisis y resultados}

El 99\% de los estudiantes que participaron en el estudio reconocieron que forman parte de, al menos, una red social. La finalidad de su pertenencia responde, según los estudiantes seleccionados, a las siguientes motivaciones: Informarse $(90 \%)$; pasar el rato o entretenerse (72\%); o encontrar amigos (44\%). Un $28 \%$ señaló que lo hacía para buscar trabajo; mientras que un 15\% indicó que su objetivo principal era buscar gente. Los porcentajes superan, en el global el $100 \%$, por que los encuestados podían seleccionar -en el marco de todas algunas preguntas del estudio- más de una casilla de respuesta. Partiendo de estos datos generales e introductorios, es posible elaborar un listado de conclusiones que permiten articular un diagnóstico general del tipo de uso y concepción que los estudiantes de Comunicación realizan de las redes sociales. Son los siguientes:

Las redes sociales de la amistad: Un alto porcentaje de encuestados (96\%) señaló que, al conectar a una red social, se relacionan con amigos. Por su parte, los contactos de tipo profesional ocupan el segundo lugar de importancia (42\%). Por último, se alude a "otros" perfiles de contacto (ítem que aglutina un 15\% del total de los resultados). De este modo, las redes sociales se consolidan como un espacio de entreteni-

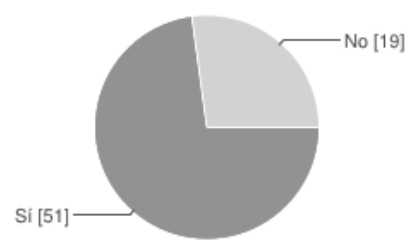

Figura 1 ¿Han cambiado las redes tu vida social?.

Fuente: elaboración propia miento donde se prima el contacto e intercambio con las amistades, por encima, de las motivaciones de tipo profesional y/o académico. A estas reflexiones se une el dato de que un $51 \%$ de los encuestados, segura que "su vida social ha cambiado con el uso de Facebook u otra red social"

Por otro lado, los resultados derivados de los focus group, ofrecen una serie de consideraciones por parte de los estudiantes que invitan a la reflexión sobre los motivos que les conducen a abrir sus cuentas en estas redes sociales. En este sentido, coinciden en señalar que el contacto con los amigos (y conocidos) constituye la motivación principal. Además, se alude a la irrupción de las redes sociales como una moda donde "se tenía que estar". A este dato se une que un $80 \%$ considera que las redes sociales "no" son una moda pasajera. 


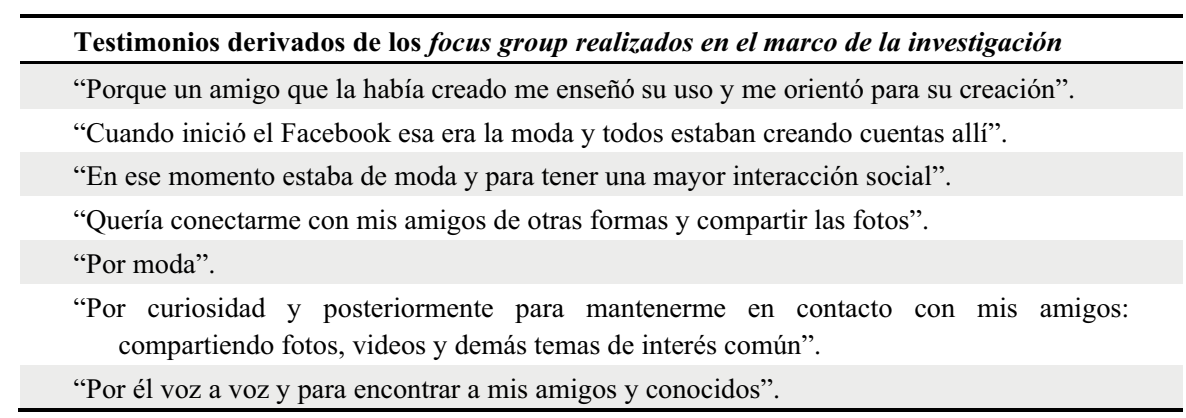

Figura 2: Motivos de la presencia en las redes sociales. Fuente: elaboración propia

A pesar de que los encuestados, no considera el establecimiento de contactos profesionales y/o académicos como el motivo de su presencia en las redes sociales, un $87 \%$ de los encuestados considera una red social como "una plataforma donde podría encontrar trabajo o trabajar".

Este dato también está ligado a la cuestión de si "estás agregado alguna red social de comunicación". Con relación a este interrogante, un 59\% señala que sí pertenece; mientras que un $39 \%$ apunta que no. En este sentido, las redes sociales de medios de comunicación más seguida por los estudiantes son las siguientes: El Colombiano, El Tiempo, La FM, La W, Caracol TV, Caracol Radio, RCN TV, RCN Radio, CNN News, El Espectador, BBC News, El País (de España).

Nuevos usuarios, ¿diferentes consumidores?: Si bien la presencia de los estudiantes en redes sociales es muy marcada, por el momento, no se han decantado por éstas -de

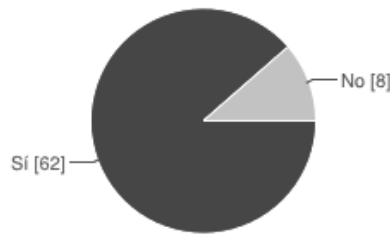

Figura 3. ¿Es una red social una plataforma donde podrías encontrar trabajo o trabajar? Fuente: elaboración propia forma mayoritaria- en detrimento de los medios de comunicación on-line. Aunque los estudiantes aseguran que su objetivo principal -al conectarse a las redes sociales- es el de informarse, se detecta una preferencia equilibrada entre informarse en cibermedio o en una red social ( $45 \%$ frente a un $46 \%$ ). No obstante, a pesar de que no se trata de una diferencia muy amplia, la cantidad de alumnos que priorizan el acceso a la in-

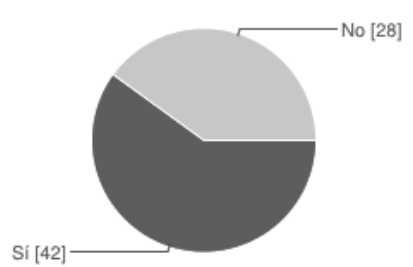

Figura 4. ¿Perteneces a alguna red social de un medio? Fuente: elaboración propia formación a través de las redes sociales es muy elevada y anticipa un cambio en el consumo -y por ende, el acceso- de los contenidos informativos en la Red.

Confusión terminológica: El rápido y mayoritario acceso de los estudiantes a las redes sociales no ha venido acompañado de una correcta asimilación de los conceptos que acompañan a esta revolución digital que ha introducido Internet y que da forma a la lla- 
mada Sociedad del Conocimiento. En este sentido, los alumnos coinciden en señalar que la principal diferencia entre un "nativo" y un "inmigrante" digital radica en aspectos cuantitativos (número de accesos a Facebook, cantidad de amigos en las redes sociales, cifra de fotografías publicadas en estas plataformas on-line, etc.). Así, algunas de las respuestas obtenidos por los participantes en el focus group sobre esta cuestión fueron "El inmigrante digital prefiere relacionarse con otras personas de un modo tradicional y aunque el nativo no deja de hacerlo, prefiere la practicidad de encontrarse en la red para agilitar las tareas" o "Los nativos saben mucho más que el inmigrante, ya hay personas que saben mucho más que yo y cada vez voy avanzando más y alejándome un poco de lo nuevo".

Una particular miradas a las fortalezas y a las debilidades: Los alumnos destacan, como principales fortalezas, los atributos informativos y comunicativos que acompañan a las redes sociales y que conforman los rasgos definitorios de los mensajes multimedia interactivos. Especialmente, destacan la celeridad que introducen en las relaciones e intercambios comunicativos. Por otro lado, al reflexionar sobre las debilidades, denotan un gran conocimiento de los riesgos que acompañan a las redes sociales, desde los delitos y faltas legales hasta el posible deterioro de la comunicación mediante rumores o informaciones no verificadas.

\begin{tabular}{|c|}
\hline Testimonios derivados de los focus group realizados en el marco de la investigación \\
\hline FORTALEZAS \\
\hline $\begin{array}{l}\text { Practicidad. Multimedia. Inmediatez. Comunicación eficiente en largas distancias. Encontrar } \\
\text { amigos y mantenerse en contacto con ellos. Rapidez. Efectividad. Oportunidad. } \\
\text { Actualidad. Interacción. Acercamiento. Se puede llamar la atención de manera más eficaz. } \\
\text { Entretenimiento. Retroalimentación. }\end{array}$ \\
\hline DEBILIDADES \\
\hline $\begin{array}{l}\text { Superficialidad. Adicción o contenidos erróneos. Terminología. Mala ortografía que se } \\
\text { adquiere. Masificación. Ciberbullyng. Creación de identidades falsas. Delitos. Rumores. } \\
\text { Información innecesaria. Chismes. Inseguridad. Sedentarismo. Genera nerviosismo. } \\
\text { Fraudes. Inseguridad. }\end{array}$ \\
\hline
\end{tabular}

Figura 5. Fortalezas y debilidades de las redes sociales. Fuente: elaboración propia

Al solicitar a los estudiantes que seleccionen un término para definir qué es una red social, aparecen un conjunto de respuestas que, de nuevo, inciden en los aspectos vinculados con la celeridad en los procesos comunicativos. Del mismo modo, se subraya el carácter útil e incluso, imprescindible que éstas poseen hoy en día. Algunos, aluden igualmente a aspectos vinculados con los riesgos. Los conceptos más utilizados son los siguientes: Novedosa, Utilidad, Indispensable, Interactividad, Compleja, Emocionante, Comunicación, Grandioso, Dinámica, Eficaz, Necesaria, Enorme, Conexión, Me gusta, Llamativa, Actual, Compartir, Adicción, Manipulable, Chisme, Rápida.

\section{Discusión y conclusiones}

En este estudio se ha analizado como estudiantes de comunicación colombianos utilizan las redes sociales y los recursos de la web 2.0 como herramientas formativas, de 
participación, colaborativas y de entretenimiento. La investigación muestra que el 99\% de los encuestados tiene perfil en alguna red social y entre uno de sus más importantes hallazgos es que su preferencia de uso es para informarse $(90 \%)$, de forma muy superior a la de relacionarse (44\%). Estos datos difieren del estudio Martínez y Torrado (2011) sobre uso de las redes sociales como fuente de información de los estudiantes de periodismo de la Universidad de Murcia. Si bien estos también tienen mayoritariamente un perfil en las redes sociales $(87 \%)$, solo un $10 \%$ destacan que las utilizan para consultar noticias y medios de comunicación. (Martínez y Torrado, 2011:363). De todas maneras, sí que ambas investigaciones coinciden en destacar que los medios de comunicación tradicionales por Internet siguen siendo la principal fuente de información a la que se dirigen los estudiantes de comunicación. En este aspecto, destacamos que se está viendo un cambio de tendencia en el consumo y que se deben seguir haciéndose análisis de este tipo porqué en un corto espacio de tiempo las redes sociales pueden convertirse en la principal fuente de información.

Sin, embargo cuando se profundiza mediante grupos de discusión vemos que estas siguen siendo básicamente un medio de relación para los estudiantes analizados, aspecto que coincide con la investigación de Pempek, et al. (2009) en relación al uso de las redes sociales por parte de los universitarios. Así, en nuestra investigación también se destaca que los estudiantes comparten contenidos con amigos con los que previamente se había establecido una relación y se usan los medios digitales como forma de expresar la propia identidad. Además, establecemos otro factor a los ya definidos por Park, et al. (2009) en su encuesta sobre Facebook dirigida a estudiantes universitarios. Si estos, identificaron cuatro necesidades básicas para conectarse a las redes: socializar, entretenimiento, búsqueda de la propia identidad e información, nosotros podemos añadir la de buscar trabajo y actividades relacionadas con el ámbito profesional, que ya fue también enunciada por Gómez, et al. (2012).

Por otro lado, la falta de asimilación de los conceptos definidos académicamente y relacionados con la nueva era digital por parte de los estudiantes de la muestra, tal y como son los términos "nativo" e "inmigrante" digital, puede estar relacionado con los hallazgos de Jones, et al. (2010) que a partir de un estudio de estudiantes de primer curso en cinco universidades inglesas, defienden que la complejidad y las diferencias en el uso de las tecnologías por parte de los alumnos, no se podría hablar de que exista una generación digital o de que los "nativos digitales" tengan unos conocimientos homogéneos diferentes a los "inmigrantes digitales".

Finalmente, este estudio demuestra que las redes sociales son un reto formativo para una nueva generación de estudiantes universitarios, no solo europeos o norteamericanos, sino también Latinoamericanos. Tal y como ellos mismos destacan, su practicidad, inmediatez y eficacia las convierten en un instrumento especialmente relevante para alumnos de comunicación. Al igual que Stornaiuolo, et al. (2013) consideramos que las redes sociales pueden ser el instrumento ideal para fomentar las competencias comunicativas en el ámbito educativo. Además, al igual que en la investigación de Bernal y Angulo (2013) sobre jóvenes andaluces, donde se afirmaba que estos tenían muy claro cuáles eran los peligros de las redes digitales, es importante tener en cuenta que los universitarios colombianos de comunicación también conocen 
cuáles son los riegos de las redes sociales y de Internet en general. Así, en los grupos de discusión han destacado aspectos tan importantes como la superficialidad, la mal ortografía que se adquiere, el ciberbullyng, los aspectos psicológicos y relacionales y los aspectos delictivos y de inseguridad. ¿Pero hasta qué punto los estudiantes universitarios actúan en las redes sociales teniendo en cuenta estos factores negativos que parecen conocer tan bien? ¿Hasta qué punto son críticos en su acceso a los contenidos digitales? Este es un ámbito en el que se debe seguir estudiando y que nos plantea nuevos retos en la investigación. Al mismo tiempo, que plantea nuevas oportunidades a los docentes y a la universidad, ámbito en el que los estudiantes de comunicación deben formarse en estos aspectos.

\section{Referencias bibliográficas}

ALLAN, Stuart \& THORSEN, Einar (Eds., 2009): Citizen Journalism: Global Perspectives. New York, Peter Lang Publishing.

BANCO MUNDIAL (2013): Datos Colombia en El Banco Mundial: http://datos.bancomundial.org/pais/colombia [Consulta: 2 de febrero de 2014].

BERNAL, César y ANGULO, Félix (2013): "Interacciones de los jóvenes andaluces en las redes sociales". Comunicar, núm. 40. Huelva, Grupo Comunicar, pp. 25-30. (DOI: 10.3916/C40-2013-02-02).

CASTRILLÓN, Gisel Lorena (2013): Redes sociales, oportunidad de aprendizaje para los jóvenes universitarios. Medellín, Trabajo de Grado. Universidad Pontificia Bolivariana (UPB).

CERLAC-UNESCO (2013): El libro en cifras. Boletín estadístico del libro en Iberoamérica. Cerlac-Unesco: Bogotá. http://cerlalc.org/wp-content/uploads/2013/07 /Libro_Cifras_3.pdf [Consulta: 15 de noviembre de 2013].

DABBAGH, Nada \& KITSANTAS, Anastasia (2012): "Personal Learning Environments, social media, and self-regulated learning: A natural formula for connecting formal and informal learning". The Internet and Higher Education, $\mathrm{n}^{\circ} 15$ (1). London, Elsevier, pp. 3-8. DOI: http://dx.doi.org/10.1016/j.iheduc.2011.06.002

DANE (2013): Encuesta de Consumo Cultural. Bogotá, Departamento Administrativo Nacional de Estadística, Dane. (http://www.dane.gov.co/files/investigaciones/eccultulral/presentacion_ecc_2012.pdf.) [Consulta: 10 de noviembre de 2013].

ELTIEMPO.COM (2013): "Directivos de la red social están en el país coordinando la llegada de la empresa" El Tiempo.com, en: http://www.eltiempo.com/tecnologia/actualidad/ARTICULO-WEB-NEW_NOTA_INTERIOR-13099656.html) [Consulta: 15 de diciembre de 2013].

GÓMEZ, Marisol; ROSES, Sergio; y FARIAS, Pedro (2012): "El uso académico de las redes sociales en universitarios". Comunicar, núm. 38, Huelva, Grupo Comunicar, pp. 131-138. DOI: 10.3916/C38-2012-03-04.

HARRISON, Teresa M. \& BARTHEL, Brea (2009): "Wielding new media in Web 2.0: exploring the history of engagement with the collaborative construction of 
media products". New Media \& Society, num. 11 (1-2), Chicago, University of Illinois, Sage Journals, pp.155-178. DOI: 10.1177/1461444808099580.

ITU (2012): Medición de la Sociedad de la Información de la Unión internacional de Telecomunicaciones. Informe ejecutivo. Ginebra, Unión Internacional de Telecomunicaciones. Suiza, Place des Nations. http://www.itu.int/ITU-D/ict/publications/idi/index.html) [Consulta: 12 de diciembre de 2013].

JENKINS, Henry (2008): Convergence Culture: Where Old and New Media Collide. New York, NYU Press.

JONES, Chris; et al. (2010): "Net generation or Digital Natives: Is there a distinct new generation entering university?" Computers \& Education, num. 54 (3), Elsevier, pp. 722-732. DOI: http://dx.doi.org/10.1016/j.compedu.2009.09.022

LEWIS, Seth C.; KAUFHOLD, Kelly; \& LASORSA, Dominic L. (2009): “Thinking about citizen journalism. The philosophical and practical challenges of user-generated content for community newspapers". Journalism Practice, num. 4 (2), Taylor \& Francis, pp. 136-179. DOI:10.1080/14616700903156919

MARTÍNEZ, Lourdes y TORRADO, Susana (2011): “Uso de las redes sociales como fuente de información de los futuros profesionales de la comunicación: ¿ilusión o realidad?", Trípodos, extra. Barcelona, Universitat Ramon Llull, pp. 359-367: http://cicr.blanquerna.url.edu/congres_vi/actas/dades/ambit2/18.\%20Mart\%C3\%A Dnez, Torrado.pdf [fecha de consulta: 10 de diciembre de 2013].

MINTIC (2011): "Locomotora de las TIC va a todo vapor". Bogotá, MINTIC Ministerio de Tecnologías de la Información y las Comunicaciones. http://www.mintic.gov.co/index.php/mn-news/714-20111221-colombia-se-subio-a-la-locomotorade-la-conectividad [Consulta: 8 de diciembre de 2013].

MINTIC (2013): Boletín trimestral de las TIC. Cifras cuarto trimestre de 2012. Colombia, MINTIC http://www.mintic.gov.co/images/documentos/cifras_del_sector/boletin_4t_banda_ancha_vive_digital_2012.pdf [Consulta: $16 \mathrm{de}$ diciembre de 2013].

O'REILLY, Tim (2007): "What is Web 2.0: Design Patterns and Business Models for the Next Generation of Software", in O'Reilly.com http://oreilly.com/web2/archive /what-is-web-20.html [Consulta: 16 de enero de 2013].

PALACIOS, Marcos y DÍAZ NOCI, Javier (Eds., 2007): Ciberperiodismo: Métodos de investigación. En: http://www.ehu.es/argitalpenak/images/stories/libros_gratuitos_en_pdf/Ciencias_Sociales/Ciberperiodismo $\% 20 \% 20$ Metodos $\% 20 \mathrm{de} \% 20 \mathrm{in}$ vestigacion.pdf [Consulta: 24 de noviembre de 2013].

PARK, Namsu; KEE, Kerk F.; \& VALENZUELA, Sebastián (2009): "Being Immersed in Social Networking Environment: Facebook Groups, Uses and Gratifications, and Social Outcomes". CyberPsychology \& Behavior, num. 12 (6), International Association of CyberPsychology, Training \& Rehabilitation (iACToR), pp. 729-733. DOI: 10.1089/cpb.2009.0003 
PEMPEK, Tiffany A.; YERMOLAYEVA, Yevdokiya A; \& CALVERT, Sandra L. (2009): "College students' social networking experiences on Facebook", Journal of Applied Developmental Psychology, Elsevier, num. 30 (3), pp. 227-238. DOI: http://dx.doi.org/10.1016/j.appdev.2008.12.010

PURCELL, Kristen; RAINIE, Lee; MITCHELL, Amy; ROSENSTIEL, Tom; \& OLMSTEAD, Kenny (2010): Understanding the participatory news consumer. How internet and cell phone users have turned news into a social experience. Pew Research Center. Disponible en línea: http://www.pewinternet.org/ /media /Files/Reports/2010/PIP_Understanding_the_Participatory_News_Consumer.pdf [Consulta: 30 de enero de 2013].

ROBLYER, Margaret D.; McDANIEL, Michelle; WEBB; Marsena; HERMAN, James; \& WITTY, James V. (2010): "Findings on Facebook in higher education: A comparison of college faculty and student uses and perceptions of social networking sites". The Internet and Higher Education, num. 13 (3), NSU, Elsevier, pp. 134-140. DOI: http://dx.doi.org/10.1016/j.iheduc.2010.03.002

STORNAIUOLO, Amy; DIZIO, Jennifer; y HELLMICH, Emmy (2013): "Desarrollando la comunidad: jóvenes, redes sociales y escuelas" Comunicar, num. 40. Huelva, Grupo Comunicar, pp. 79-88. DOI: 10.3916/C40-2013-02-08

THE COCKTAIL ANALYSIS (Ed., 2013): Informe de resultados: $5^{a}$ Oleada Observatorio de Redes Sociales. http://tcanalysis.com/ [Consulta: 24 de noviembre de 2013].

THURMAN, Neil (2008): "Forums for citizen journalists? Adoption of user generated content initiatives by online news media". New Media \& Society, num. 10 (1), Chicago, University of Illinois, Sage Journals, 139-157. DOI: http://dx.doi.org/10.1177/1461444807085325

TUÑEZ, Miguel; MARTÍNEZ, Yolanda; y ABEJÓN, Paloma (2010): "Nuevos entornos, nuevas demandas, nuevos periodistas" Estudios sobre el Mensaje Periodístico, vol. 16, pp. 79-94. Madrid, Servicio de Publicaciones de la Universidad Complutense.

UIS (2013): Adult and Youth Literacy. En: http://www.uis.unesco.org/literacy/Documents/fs26-2013-literacy-en.pdf [Consulta: 24 de noviembre de 2013].

WAYCOTT, Jenny; BENNETT, Sue; KENNEDY, Gregor; DALGARNO, Barney; \& GRAY, Kathleen (2010): "Digital divides? Student and staff perceptions of information and communication technologies". Computers \& Education, num. 54 (4), Elsevier, pp. 1202-1211. DOI: http://dx.doi.org/10.1016/j.compedu.2009.11.006 